\title{
Management Options for Infertile Women with Polycystic Ovary Syndrome
}

\author{
Giulia Mariani' and José Bellver ${ }^{1,2}$
}

1. Instituto Valenciano de Infertilidad, University of Valencia, Spain; 2. Department of Pediatrics, Obstetrics and Gynecology, Faculty of Medicine, University of Valencia, Spain

$\mathrm{P}$ olycystic ovary syndrome (PCOS) is a heterogenous condition affecting $5-10 \%$ of women at reproductive age. It is the most common cause of anovulatory infertility, characterized by hyperandrogenism and arrested follicle development, and is frequently associated with metabolic features such as insulin resistance and obesity. The diagnosis of PCOS, based on the Rotterdam criteria (2003), can be made when at least two of the following three main features are met: oligo-ovulation and/or anovulation, hyperandrogenism (clinical and/or biochemical), and polycystic ovarian morphology at ultrasound examination. Several approaches to ovulation induction have been proposed in women with PCOS. These approaches vary in efficacy, treatment duration, cost, and patient compliance. Management includes lifestyle changes, pharmacotherapy (metformin, clomiphene citrate, letrozole, gonadotropins, inositol), laparoscopic surgery (ovarian drilling), and assisted reproductive techniques, usually in vitro fertilization (see Figure 1). Clinical decisions in PCOS anovulatory patients are currently supported by a recently published international evidence-based guideline that provides 166 recommendations to help clinicians in the diagnosis and management of PCOS and to guide clinical practice.

\section{Keywords}

Polycystic ovary syndrome (PCOS), anovulatory infertility, ovulation induction, lifestyle, clomiphene citrate, metformin, letrozole, gonadotropins, ovarian drilling, in-vitro fertilization (IVF)

Disclosures: Giulia Mariani and José Bellver Pradas have no relevant conflicts of interest to declare.

Review Process: Double-blind peer review.

Authorship: All named authors meet the criteria of the International Committee of Medical Journal Editors for authorship for this manuscript, take responsibility for the integrity of the work as a whole and have given final approval for the version to be published.

open Access: This article is published under the Creative Commons Attribution Noncommercial License, which permits any non-commercial use, distribution, adaptation and reproduction provided the original author(s) and source are given appropriate credit. (C) The Authors 2018.

Received: June 20, 2018

Accepted: September 12, 2018

Citation: US Endocrinology. 2018;14(2):67-72

Corresponding Author: Giulia Mariani, MD, Instituto Valenciano de Infertilidad, University of Valencia, Plaza de la Policía Local 3, 46015, Valencia, Spain E: giulia.mariani@ivirma.com

Support: No funding was received fo the publication of this manuscript.

\section{Lifestyle}

Obesity has a negative impact on the reproductive outcome of women with polycystic ovary syndrome (PCOS). It affects natural conception reducing the chance of spontaneous ovulation, and increases the rate of miscarriage as well as the risk for complications during pregnancy and adverse perinatal outcomes. ${ }^{1-4}$ Lifestyle modification programs based on a combination of hypocaloric diet, increased physical activity, and behavioral interventions have shown a significant improvement in metabolic and anthropometric features (body weight, waist-hip ratio, and body composition) in women with PCOS. ${ }^{5}$ Furthermore, the reduction of excessive levels of insulin and androgens in blood leads to the restoration of menstrual regularity and ovulation, improving the reproductive function in PCOS infertile women. ${ }^{6}$

Diet with regular exercise should be recommended as first-line therapy for weight loss in overweight or obese women with PCOS (body mass index [BMI] $>25 \mathrm{~kg} / \mathrm{m}^{2}$ ). ${ }^{7}$ However, it seems to be beneficial for all women with PCOS, regardless of their weight and even when no weight loss occurs, as it may improve metabolic risk factors associated with PCOS. ${ }^{8}$ Overweight women with PCOS should lose at least $5-10 \%$ of their initial weight to experience significant health benefits, including spontaneous ovulation. ${ }^{9}$ Lifestyle interventions consist of a hypocaloric diet (500-1,000 calorie deficit per day) in combination with physical exercise (20-60 minutes of exercise per day from 3-5 times/week for 6 months). ${ }^{10,11}$ This is a cost-effective initial treatment strategy compared with surgical and medical options. If the patient's compliance with lifestyle is limited, pharmacotherapy can be considered. When metformin is associated with lifestyle for a period of at least 6 months, a greater, but not statistically significant reduction, in BMI has been shown, with significantly lower subcutaneous fat and improved menstrual cyclicity compared with lifestyle + placebo. ${ }^{12}$

\section{Bariatric surgery}

Current guidelines suggest that bariatric surgery should be considered in obese women with a $\mathrm{BMI} \geq 40 \mathrm{~kg} / \mathrm{m}^{2}$ or $\geq 35 \mathrm{~kg} / \mathrm{m}^{2}$ with associated comorbidities who have failed to lose weight with a lifestyle management program of at least 6 months. ${ }^{13}$ This surgery is not only an effective treatment in inducing and maintaining weight loss, but it could also improve fertility outcomes in obese women with PCOS, restoring regular menstrual cycles and ovulation, reducing hyperandrogenism and insulin resistance, and favoring sexual activity. ${ }^{14,15}$ Patients must be made aware of the risks of this surgeryrelated to the surgery itself or to the complications during pregnancy and the neonatal period. 
Figure 1: Flow chart for ovulation induction in infertile polycystic ovary syndrome women

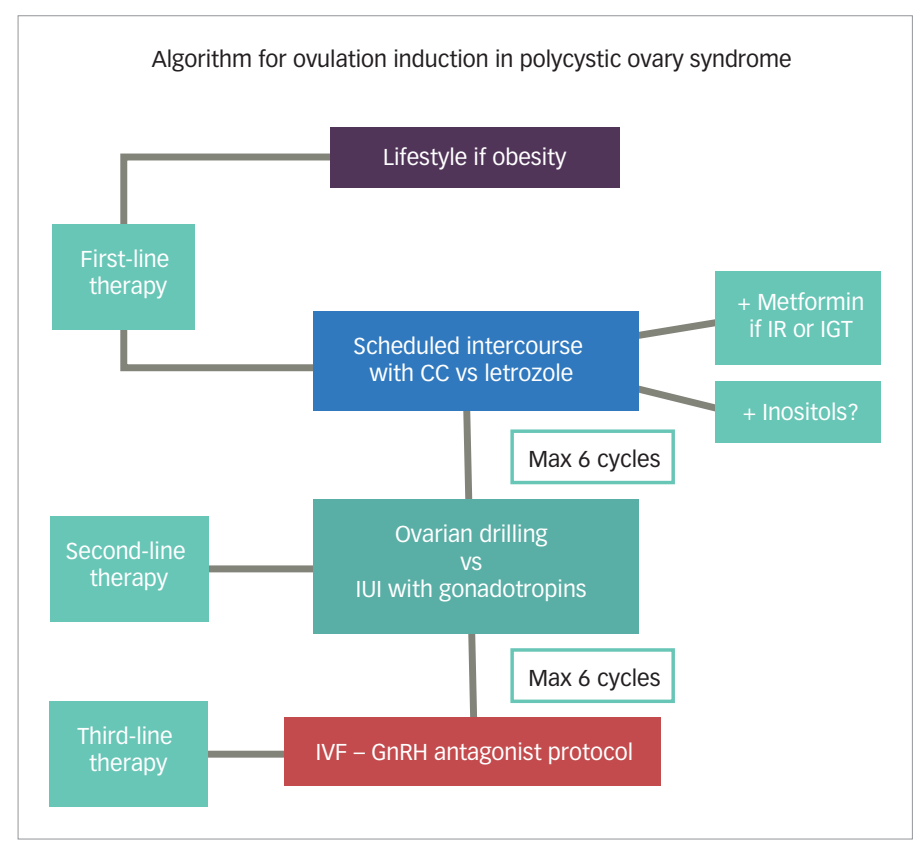

$C C=$ clomiphene citrate $; G R H=$ gonadotropin-releasing hormone; $I G T=$ impaired glucose tolerance; $I R=$ insulin resistance; $I U I=$ intrauterine insemination; IVF = in vitro fertilization.

In fact, whereas the risk for gestational diabetes and large-for-gestational age infants is reduced, the risk for small-for-gestational age infants, shorter gestation, and stillbirth or neonatal death is increased. ${ }^{16}$ For these reasons, a pregnancy achieved after bariatric surgery should be considered high risk, requiring careful monitoring of fetal growth. Furthermore, patients should wait at least 12-18 months after surgery before becoming pregnant. ${ }^{15}$ This will reduce fetal complications and to overpass this initial period of rapid weight loss with nutritional deficiencies. ${ }^{17}$ Therefore, bariatric surgery should be considered the last therapeutic option in PCOS obese women with infertility. The greater the aggressiveness of the surgery, the greater the effect in reducing weight, but the greater the risk there will be for the patient as well as for her future pregnancy.

\section{Metformin}

Metformin, a synthetically derived biguanide, is the first-line oral hypoglycemic treatment for type 2 diabetes mellitus. It reduces hepatic glucose production, intestinal glucose uptake, and increases peripheral glucose uptake by skeletal muscle and liver. As an insulin-sensitizing agent it is used in patients with PCOS to reduce serum insulin concentrations and therefore improve the metabolic features of this syndrome and treat PCOS-related anovulation. The starting dose is $500 \mathrm{mg}$ per day with a gradual increase up to $850-1,000$ mg twice daily to manage its common gastrointestinal side effects.

Although lifestyle modification is the first-line management in PCOS obese women with infertility, metformin may be useful in weight reduction. A recent systematic review and meta-analysis including 12 studies with 608 participants has shown that the combination of metformin with lifestyle is more effective in weight loss than lifestyle alone in patients with
PCOS. ${ }^{12}$ Six months of lifestyle + metformin (1.5-2 g daily) are associated with a lower BMl compared with lifestyle + placebo. When considering metformin alone versus placebo or no treatment, no effect has been shown on BMI. ${ }^{18}$ Lifestyle + metformin combined also improve menstrual cycle frequency compared with lifestyle + placebo. ${ }^{12}$ The latest update of the Cochrane review, which includes 42 trials investigating the benefit of metformin in women with PCOS, has shown higher ovulation and clinical pregnancy rates in the metformin arm compared with placebo. ${ }^{19}$

However, there is a lack of good quality evidence to support a benefit of metformin on the live birth rate. This review update includes a welldesigned Finnish multicenter, double-blind, placebo-controlled trial of 320 women, who randomly received metformin, $1.5 \mathrm{~g} /$ day (nonobese women) or $2 \mathrm{~g}$ /day (obese women), or placebo for 3 months. ${ }^{20}$ If pregnancy occurred after the third month with or without another infertility treatment combined, metformin/placebo was continued up to 12 weeks of gestation. This study showed an increase in pregnancy rate from $40.4-53.6 \%(p \leq 0.006)$ and in live birth rate from $28.8-41.9 \%(p \leq 0.014)$ in women taking metformin, with the most beneficial effects observed in obese women. Therefore, 3 months pretreatment with metformin followed by the addition of another ovulation-inducing drug may increase the live birth rate in infertile women with PCOS.

Although metformin alone compared with placebo increases the ovulation rate in women with PCOS, it should not be used as first-line therapy for anovulation. ${ }^{21}$ This is because oral ovulation induction agents, such as clomiphene citrate (CC) or letrozole alone, are more effective in the achievement of ovulation, clinical pregnancy, and live birth in women with PCOS. ${ }^{18,22}$

Metformin has also been evaluated in combination with CC. The cotreatment with CC may be beneficial for women who are resistant to CC. A Dutch meta-analysis has demonstrated that the combined administration of $\mathrm{CC}+$ metformin significantly increases the clinical pregnancy rate (relative risk [RR] 5.6; 95\% confidence interval [Cl] 2.3-13) and live birth rate (RR $6.4 ; 95 \% \mathrm{Cl} 1.2-34$ ) compared with CC alone in CCresistant women..$^{23}$ However, the latest update of the Cochrane review provides only low-quality evidence to support the increase in the live birth rate in patients receiving CC and metformin combined..$^{19}$

A recent meta-analysis included 10 randomized controlled trials (RCTs) with a total of 845 infertile women with PCOS undergoing in vitro fertilization/ intracytoplasmic sperm injection (IVF/ICSI) treatment. ${ }^{24}$ The authors found that metformin has no clinical effect on the rate of pregnancy or live birth, but reduces the risk for ovarian hyperstimulation syndrome (OHSS). A later meta-analysis, including 12 RCTs and 1,516 patients, has shown the same results: metformin does not improve assisted reproductive technology outcomes among infertile patients with PCOS. ${ }^{25}$ The only benefit deriving from the use of metformin was the decrease in the risk for OHSS.

In conclusion, metformin represents the main insulin sensitizing agent in the management of infertile women with PCOS, with no clear benefit in improving live birth rates. It is a low-cost therapy that does not require monitoring and does not induce multiple pregnancies. ${ }^{26}$ Furthermore, it has a safety profile: no evidence of teratogenity has been demonstrated when used in the first trimester of pregnancy and no short-term adverse effects have been shown on pregnancy. ${ }^{27-29}$ Only limited information 
exists about long-term effects in children exposed to metformin in utero. ${ }^{30}$ Even though metformin appears to be safe during pregnancy, there is no indication to continue its use until delivery in women with PCOS as it does not reduce pregnancy complications. ${ }^{31}$ Gastrointestinal symptoms, such as diarrhea, vomiting, nausea, and abdominal disconfort, are frequent in patients receiving metformin and are a common cause of treatment discontinuation. ${ }^{20,32}$

\section{Clomiphene citrate}

$\mathrm{CC}$ is a selective estrogen receptor modulator that competes for receptorbinding sites with endogenous estrogens. Its anti-estrogenic effect leads to an alteration in cervical function and endometrial receptivity that might impair implantation after successful induction of ovulation. It is generally considered the first-line treatment for anovulatory women with PCOS. The starting dose is $50 \mathrm{mg} /$ day for 5 days (day $3-7$ of the cycle), and it may be increased by $50 \mathrm{mg}$ a day up to a maximum of $150 \mathrm{mg} /$ day. However, clomiphene resistance (failure to achieve ovulation after a dose of $150 \mathrm{mg}$ CC per day for 5 days) is a relatively common event, occurring in approximately $15-40 \%$ of women with PCOS. ${ }^{33}$ The ovulation rate in women with PCOS after CC administration is approximately $75-80 \%$, whereas the pregnancy rate is $22 \%$ by ovulatory cycle. ${ }^{34}$ This discrepancy of around $40 \%$ between the ovulation rate and the pregnancy rate was mainly related to the hypoestrogenic effect of $\mathrm{CC}$ on the endometrium and cervical mucus. $\mathrm{Cc}$ treatment should be limited to six ovulatory cycles, as the cumulative pregnancy rate among anovulatory women with PCOS is about $65 \%$ after six CC cycles. ${ }^{35}$

In a large metanalysis, ${ }^{36}$ including 57 trials and 8,082 women, the authors have compared all of the most common regimens of ovulation induction with each other. All pharmacological treatments $(\mathrm{CC}$, metformin, $\mathrm{CC}+$ metformin, letrozole, tamoxifen, ovarian drilling, follicle stimulating hormone [FSH]) were superior to placebo or no intervention in terms of pregnancy and ovulation rates, so expectant management is not recommended in anovulatory patients with PCOS. Compared with letrozole, clomiphene alone showed lower ovulation, pregnancy, and live birth rates. A recent systematic review ${ }^{37}$ analyzing the impact of CC and other ovulationinduction drugs on endometrial thickness in women with ovulatory disorders confirmed these results. Maybe due to the lower endometrial thickness associated, CC resulted in lower pregnancy and live birth rates than letrozole for comparable ovulation rates.

Lower fertility outcomes have also been obtained using CC compared with gonadotropins. The latest Cochrane review about this topic ${ }^{38}$ has shown that CC may reduce the chance of live birth or ongoing pregnancy in terms of gonadotropins. Although the live birth rate is higher, gonadotropins are considered a second-line intervention in anovulatory women with PCOS due to the greater probability of multiple pregnancy, the increased risk for OHSS, and their higher cost. CC is still the drug most frequently used worldwide for ovulation induction in patients with PCOS. ${ }^{34,39}$ It is administered orally, is cheap, effective, safe on offspring, with few side effects. ${ }^{40}$ Among its drawbacks, a long half-life (2 weeks) that results in long-lasting adverse effects on cervical mucus and endometrial development, and the need for careful monitoring to minimize the risk for multiple pregnancy. ${ }^{26}$

If the patient does not get pregnant after the use of $\mathrm{CC}$, gonadotropins for timed intercourse or ovarian drilling are the next steps to manage anovulatory infertile women with PCOS.

\section{Letrozole}

Letrozole is the most commonly used aromatase inhibitors (Als) for ovulation induction, representing an alternative to CC treatment, especially in those women resistant to $\mathrm{CC}$. The mechanism of action consists of blocking the conversion of androgens to estrogens in the ovarian follicles, peripheral tissues, and the brain. As there is no estrogen receptor antagonism, antiestrogenic effects on the endometrium and cervical mucus are not expected, and normal negative feedback occurs generally leading to a single dominant follicle growth and monoovulation. The starting dose for letrozole is $2.5 \mathrm{mg}$ a day for 5 days from day 3-7 of the cycle. The dose is increased by $2.5 \mathrm{mg}$ a day up to a maximum daily dose of $7.5 \mathrm{mg}$ for 5 days.

The superiority of letrozole to CC in terms of ovulation, pregnancy, and live birth rate has been shown in several clinical trials.36,41,42 A large double-blind multicenter study, including 750 infertile women with PCOS randomly assigned to receive either letrozole (up to $7.5 \mathrm{mg}$ /day for 5 days) or CC (up to $150 \mathrm{mg} /$ day for 5 days) for up to five menstrual cycles, has demonstrated the greater efficacy of letrozole versus CC for ovulation induction. ${ }^{43}$ Women who received letrozole had a significantly higher ovulation rate (61.7 versus $48.4 \%$; $p<0.001$ ) and a higher cumulative live birth rate (27.5 versus $19.1 \% ; p=0.007$ ) compared with women who received CC for all BMIs, with no differences among miscarriage or twinning rates. In the subgroup of women with BMI $<30 \mathrm{~kg} / \mathrm{m}^{2}$, both treatments were equally effective in terms of live birth rate. Similarly, a recent Cochrane review ${ }^{44}$ including nine RCTs and 1,783 participants has shown increased clinical pregnancy and live birth rates using letrozole versus CC. However, no recommendation was given by the authors because the quality of the evidence was considered low. These better fertility outcomes could in part be explained through the fewer adverse effects of letrozole on oestrogen target tissues, such as endometrium and cervix, and due to the letrozole short half-life elimination time of 48 hours compared with CC. ${ }^{45}$

In conclusion, letrozole has been suggested as a possible better option than clomiphene for ovulation induction, especially in obese women with PCOS, although it is still an off-label drug in many countries.

\section{Ovarian drilling}

Laparoscopic ovarian drilling (LOD) can be used as a second-line treatment in CC-resistant women with PCOS. ${ }^{34}$ The physiopathological theory explaining the mechanism underlying ovulation induction in patients undergoing ovarian drilling is that the partial destruction of the ovarian cortex causes a local and systemic reduction in androgens, a fall in luteinizing hormone $(\mathrm{LH})$ and an increase in FSH levels. These endocrine changes lead to the promotion of follicular recruitment, maturation, and subsequent ovulation. ${ }^{46}$ Ovarian drilling is commonly performed using either heat (monopolar or bipolar electrocautery) or laser with comparable outcomes. ${ }^{47}$ However, the most used technique is to perform three to eight perforations on the surface of each ovary using monopolar energy leading to further normal ovulation in $74 \%$ of the cases in the next 3-6 months. ${ }^{48}$ Several changes to the original technique have been proposed over the years.

A recent meta-analysis, ${ }^{49}$ which included eight studies with 484 participants, compared the effectiveness of unilateral versus bilateral ovarian drilling in improving fertility outcomes in infertile women with PCOS resistant to CC. No significant difference was shown not only in terms of ovulation, pregnancy, live birth, or miscarriage rates between these two techniques, but also not even in serum anti-Müllerian hormone $(\mathrm{AMH})$ concentration 6 months after surgery. Therefore, ovarian drilling cortical damage does not seem to 
have a negative impact on ovarian reserve, although this result should be interpreted with caution due to the heterogeneity of the included studies.

The main benefit of this minimally invasive surgery compared with medical therapy is that it reduces the risk for multiple pregnancy or OHSS without decreasing the rate of pregnancy. In the last Cochrane review about this topic $^{50}$ there was no difference in live birth rate when LOD was compared with gonadotropins or with Als. Several factors could influence the efficacy of ovarian drilling. A recent review has shown that obesity, long duration of infertility $>3$ years, low basal LH levels $<10 \mathrm{IU} / \mathrm{I}$, testosterone level $>4.5 \mathrm{nmol} / \mathrm{L}$, and high basal $\mathrm{AMH}>7.7 \mathrm{ng} / \mathrm{mL}$ are surgery success predictors associated with poor response to LOD. ${ }^{51}$

Considering the comparable efficacy of LOD to other induction agents, this type of surgery may be an attractive option for infertile women with PCOS resistant to CC or letrozole, especially if laparoscopy is indicated for other reasons such as tubal patency assessment. The risks of LOD are all those related to a surgical abdominal procedure, including the formation of postoperative periovarian adhesions ${ }^{52}$ and a hypothetical reduction in ovarian reserve secondary to excessive ovarian damage. ${ }^{53}$ This continues to represent a controversial topic as most of the changes in the ovarian reserve markers observed after LOD could be interpreted as a normalisation of ovarian function rather than a reduction of ovarian reserve.

In conclusion, before referring a patient for ovarian drilling surgery, it is always necessary to evaluate the prognostic factors and carry out a riskbenefit cost balance.

\section{Gonadotropins}

Gonadotropins (FSH or human menopausal gonadotropin [HMG]) represent the second-line of medical treatment in women with PCOS who did not ovulate or conceive with $\mathrm{CC}$ and letrozole. Older anovulatory patients with PCOS could benefit from the use of a first-line treatment with gonadotropins. ${ }^{54}$

The mechanism of action of exogenous gonadotropins is to increase FSH levels in women with PCOS and stimulate follicular growth. The recommended starting dose employed to avoid hyperresponse is between 37.5-75 IU/day. ${ }^{34}$ A low-dose step-up protocol with small increases every 7 days according to follicular growth is indicated in case of no response after 14 days of ovarian stimulation. The identification of the optimal dose to be administered may require more than one ovulation induction cycle. Dosage is reduced if excessive follicular growth and/or estradiol (E2) not in accordance with follicular growth are observed. A single injection of $250 \mu \mathrm{g}$ human chorionic gonadotropin (hCG)-r of 5,000 IU hCG-u is usually used to trigger ovulation when at least one or two follicles of at least $18 \mathrm{~mm}$ in their maximum diameter have developed. In the case of multifollicular growth (three or more follicles $>14 \mathrm{~mm}$ in diameter) hCG should not be administered to prevent the risks for multiple pregnancy and OHSS. The treatment should be repeated up to a maximum of six cycles. With the low-dose step-up regimen the monofollicular ovulation rate is nearly $70 \%$, whereas the pregnancy rate is $20 \%$ per cycle. ${ }^{34}$ Low-dose step-up and low-dose step-down protocols are more effective than the step-down conventional protocol, and both have the same pregnancy rate per started cycle. ${ }^{55}$

In anovulatory women hyperresponding to FSH during ovulation induction, the addition of recombinant LH (rLH) to FSH in the late follicular phase may lead to the dominance of a single follicle, inducing the atresia of secondary follicles. ${ }^{56}$ In fact, LH activity in menotrophin preparations for ovulation induction seems to modify follicular development, leading to a significant reduced number of intermediate-sized follicles. ${ }^{57}$

A recent Cochrane review, comparing the effectiveness of different gonadotropin preparations for ovulation induction in women with PCOS resistant to $\mathrm{CC}$, has shown no difference in live birth rates and OHSS incidence using urinary FSH, recombinant FSH, or HMG/highly purifiedHMG, although the quality of evidence was low or very low. ${ }^{58}$ Thus treatment outcomes depend more on the administered dose of gonadotrophins than on the preparation used..$^{59}$

The combined therapy with metformin has shown good fertility results. Metformin co-treatment may improve the effectiveness of ovulation induction with gonadotrophins, increasing live birth rates compared with gonadotropins alone (odds ratio [OR] 2.46, 95\% Cl 1.36-4.46).60

Although time to pregnancy is shorter and pregnancy and live birth rates are higher after ovulation induction with low-dose FSH than with CC administration, gonadotropins are more expensive and have more adverse effects (OHSS, multiple pregnancies) than CC, so we always should balance the pros and cons of using one drug over another. In addition to the high cost, the other main drawback is the need for laboratory and ultrasound monitoring performed by a specialist.

\section{In vitro fertilization}

Ovarian stimulation and IVF are considered the third-line treatment for infertile women with PCOS. IVF is especially recommended if there are additional infertility factors, such as tubal damage, advanced woman age, severe endometriosis, and male subfertility. ${ }^{34}$ A single embryo-transfer procedure markedly reduces the risk for multiple pregnancy, which is one of the main drawbacks of using gonadotropins.

Despite a higher cycle cancellation rate in patients with PCOS, clinical pregnancy and the live birth rates are comparable to those of women without PCOS. ${ }^{61,62}$ The use of gonadotropin-releasing hormone (GnRH) antagonist limits the occurence of OHSS, whose incidence is significantly higher in women with PCOS (15\%) compared with those with normal ovaries (3\%). ${ }^{63}$ Women treated with the GnRH antagonist "short protocol" have a lower incidence of OHSS compared with those receiving the "Iong GnRH agonist regimen" (OR 0.61, 95\% C 0.51-0.72; 36 RCTs, $n=7,944$ ) without reducing the likelihood of achieving live birth (OR 1.02, 95\% Cl 0.85-1.23; 12 RCTS, $n=2,303) .{ }^{64}$

Another important advantage of the GnRH antagonist protocol is that it allows the final oocyte maturation with a bolus of GnRH agonist to be triggered instead of hCG, a procedure that significantly reduces or totally eliminates the risk for OHSS in high-risk patients. GnRH-agonist administration for final oocyte maturation is associated with a luteal phase defect that leads to a significantly reduced likelihood of ongoing pregnancy achievement compared with standard hCG treatment. ${ }^{65}$ No difference regarding the number of oocytes retrieved, the proportion of metaphase II oocytes, the fertilization rate, and embryo scores were found when hCG and GnRH agonist were compared. ${ }^{66}$ After $\mathrm{GnRH}$ agonist triggering, the best option is to freeze all embryos and transfer them in a subsequent frozen-thawed embryo replacement or natural cycle. ${ }^{67}$ Nowadays, the combined use of a $\mathrm{GnRH}$ antagonist protocol with $\mathrm{GnRH}$ agonist triggering and embryo 
freezing should be recommended as a standard preventive measure of OHSS in patients with risk for ovarian stimulation hyperresponse.

\section{Inositol}

Inositol is a sugar alcohol with multiple stereoisomers. Inositols are involved in the postreceptorial signal transmission of different hormones such as insulin, TSH, and FSH, and their metabolites have a relevant physiological role in reducing insulin resistance and hyperandrogenism, and have beneficial effects on reproductive function, ${ }^{68-72}$ so inositol supplementation has been proposed to improve reproductive outcomes in infertile women with PCOS.

The two most commonly studied isomers, myoinositol (MI) and d-chiroinositol (DCl), control key enzymes involved in glucose and lipid metabolism. DCl is mainly involved in glucose uptake and glycogen synthesis (liver, fat, and muscle), while $\mathrm{Ml}$ is responsible for the activation of gluco-transporters and glucose utilization. Ml is more beneficial in improving the metabolic profile; $\mathrm{DCl}$ is more effective in reducing androgen levels and modifying endocrine parameters. ${ }^{73}$ Treatment with $\mathrm{Ml}$ is effective not only in improving insulin resistance but it has also shown a significant advantage in terms of reduction of $\mathrm{BMI}^{74,75}$ The probability of ovulation and the chance of pregnancy are reduced in obese women with PCOS treated with MI compared with normaloverweight patients, and no metabolic benefits of Ml treatment have been observed in obese patients with a BMI $>37.75,76$ However, an Italian study has shown that $\mathrm{Ml}$ administration is more effective in those obese patients who have high fasting insulin plasma levels, ${ }^{77}$ whereas DCl administration has better results in those obese hyperinsulinemic patients with PCOS who have a history of relatives with diabetes. ${ }^{78}$

A recently published systematic review and metaanalysis of 10 RCTs including 601 women with $\mathrm{PCOS}^{79}$ has shown that inositol supplementation increases ovulation rate and menstrual cycle frequency compared with placebo. Whether used alone or in combination with $\mathrm{CC}$ in MI-resistant patients, MI produces very good clinical results, with an ovulation rate of $61.7 \%$ and $72.2 \%$, respectively, when administered during three spontaneous menstrual cycles. ${ }^{75}$ However, these results do not seem to translate into better reproductive outcomes. In the last review about inositol supplementation in women with PCOS undergoing ICSI, the authors concluded that MI does not improve oocyte or embryo quality and pregnancy rates, and that DCI has a controversial role. ${ }^{80}$ When administered, a recent international consensus conference recommends using a combination of both inositol isoforms in their physiological plasma ratio 40:1 to have benefits at systemic and ovarian levels. ${ }^{81}$

Recently, alpha-lipoic acid treatment, alone or in combination with $\mathrm{Ml}$ or $\mathrm{DCl}$, has shown to improve the metabolic parameters of obese patients with PCOS modulating glucose utilization through the activation of adenosine monophosphateactivated protein kinase in skeletal muscles thus increasing glucose-transporter-4. ${ }^{82}$ Data from Genazzani's group demonstrated a significant decrease in insulin, glucose, BMl, and Homeostasis Model Assessment index parameters after 12 weeks of daily intake of $400 \mathrm{mg}$ of alpha-lipoic acid. ${ }^{83}$

\section{Conclusion}

Different treatment options exist to achieve ovulation in women with PCOS. The clinical decision of which treatment recommend should be made on local facilities, adverse effects, cost, and patient compliance. Ovulation induction has to be individualized according to the patient weight and PCOS phenotype, with the aim of achieving mono-ovulation and subsequently the birth of a singleton healthy baby, minimizing the risks for OHSS, and multiple pregnancy. $\square$
1. Norman RJ, Dewailly D, Legro RS. Polycystic ovary syndrome. Lancet. 2007:370:685-97.

2. Rotterdam ESHRE/ASRM-Sponsored PCOS Consensus WorkshopGroup. Revised 2003 consensus on diagnostic criteria and long-term health risks related to polycystic ovary syndrome Fertil Steril. 2004;81:19-25

3. Teede HJ, Misso ML, Costello MF, et al. International PCOS Network. Recommendations from the international evidencebased guideline for the assessment and management of polycystic ovary syndrome. Fertil Steril. 2018;110:364-79.

4. Cedergren MI. Maternal morbid obesity and the risk of adverse pregnancy outcome. Obstet Gynecol. 2004;103:219-24.

5. Moran LJ, Hutchison SK, Norman RJ, Teede HJ. Lifestyle changes in women with polycystic ovary syndrome. Cochrane Database Syst Rev. 2011;7:CD007506.

6. Qublan HS, Yannakoula EK, Al-Qudah MA, El-Uri Fl. Dietary intervention versus metformin to improve the reproductive outcome in women with polycystic ovary syndrome. A prospective comparative study. Saudi Med J. 2007:28:1694-9.

7. Balen AH, Morley LC, Misso M, et al. The management of anovulatory infertility in women with polycystic ovary syndrome: an analysis of the evidence to support the development of globa WHO guidance. Hum Repr Update. 2016;22:687-708.

8. Poehlman ET, Dvorak RV, DeNino WF, et al. Effects of resistance training and endurance training on insulin sensitivity in nonobese young women: a controlled randomized trial. J Clin Endocrinol Metab. 2000;85:2463-8.

9. Radon PA, MCMahon MJ, Meyer WR. Impaired glucose tolerance in pregnant women with polycystic ovary syndrome. Obstet Gynecol. 1999;94:194-7.

10. Tang T, Glanville J, Hayden CJ, et al. Combined lifestyle modification and metformin in obese patients with polycystic ovary syndrome. A randomized. placebo-controlled. double-blin multicentre study. Hum Reprod. 2006;21:80-9.

11. Karimzadeh MA, Javedani M. An assessment of lifestyle modification versus medical treatment with clomiphene citrate. metformin. and clomiphene citrate-metformin in patients with polycystic ovary syndrome. Fertil Steril. 2010;94:216-20.

12. Naderpoor N, Shorakae S, de Courten B, et al. Metformin and lifestyle modification in polycystic ovary syndrome: systematic review and meta-analysis. Hum Reprod Update. 2015;21:560-74

13. Edinburgh Scottish Intercollegiate Guidelines Network. Management of Obesity: a National Clinical Guideline. 2010
14. Teitelman M, Grotegut CA, Williams NN, Lewis JD. The impact of bariatric surgery on menstrual patterns. Obes Surg. 2006:16:1457-63.

15. Malik SM, Traub ML. Defining the role of bariatric surgery in polycystic ovarian syndrome patients. World I Diabetes. 2012;3:71-9

16. Johansson K, Cnattingius S, Näslund I, et al. Outcomes of pregnancy after bariatric surgery. N Eng/ I Med. 2015;372:814-24.

17. Colquitt JL, Pickett K, Loveman E, Frampton GK. Surgery for weight Ioss in adults. Cochrane Database Syst Rev. 2014;8:CD003641.

18. Tang T, Lord JM, Norman RJ, et al. Insulin-sensitising drugs (metformin, rosiglitazone, pioglitazone, D-chiro-inositol) for women with polycystic ovary syndrome, oligo amenorrhoea and subfertility. Cochrane Database Syst Rev. 2012;5:CD003053.

19. Morley $L C$, Tang $T$, Yasmin $E$, et al. Insulin-sensitising drugs (metformin, rosiglitazone, pioglitazone, D-chiro-inositol) for women with polycystic ovary syndrome, oligo amenorrhoea and subfertility. Cochrane Database Syst Rev. 2017:11:CD003053.

20. Morin-Papunen L, Rantala AS, Unkila-Kallio L, et al. Metformin improves pregnancy and live-birth rates in women with polycystic ovary syndrome (PCOS): a multicenter. double-blind. placebo-controlled randomized trial. J Clin Endocrinol Metab. 2012;97:1492-500

21. Practice Committee of the American Society for Reproductive Medicine. Role of metformin for ovulation induction in infertile patients with polycystic ovary syndrome (PCOS): a guideline. Fertil Steril. 2017:108(3).

22. Siebert TI, Viola MI, Steyn DW, Kruger TF. Is metformin indicated as primary ovulation induction agent in women with PCOS? A systematic review and meta-analysis. Gynecol Obstet Invest. 2012;73:304-13.

23. Moll $\mathrm{E}$, van der Veen $\mathrm{F}$, van Wely $\mathrm{M}$. The role of metformin in polycystic ovary syndrome: a systematic review. Hum Reprod Update. 2007:13:527-37.

24. Palomba S, Falbo A, La Sala GB. Effects of metformin in women with polycystic ovary syndrome treated with gonadotrophins for in vitro fertilisation and intracytoplasmic sperm injection cycles: a systematic review and meta-analysis of randomised controlled trials. BJOG. 2013;120:267-76.

25. Huang $X$, Wang $P$, Tal $R$, et al. A systematic review and metaanalysis of metformin among patients with polycystic ovany syndrome undergoing assisted reproductive technology procedures. Int J Gynaecol Obstet. 2015;131:111-6.
26. Teede HJ, Miss ML, Deeks AA, et al. Guideline Development Groups. Assessment and management of polycystic ovary syndrome: summary of an evidence-based guideline. Med J Aust. 2011:195:S65-112.

27. Cassina M, Dona M, Di Gianantonio E, et al. First-trimester exposure to metformin and risk of birth defects: a systematic review and meta-analysis. Hum Reprod Update. 2014;20:656-69.

28. Given JE, Loane M, Garne E, et al. Metformin exposure in first trimester of pregnancy and risk of all or specific congenital anomalies: exploratory case-control study. BMJ. 2018;361:K2477.

29. Butalia S, Gutierrez L, Lodha A, et al. Short- and long-term outcomes of metformin compared with insulin alone in pregnancy: a systematic review and metaanalysis. Diabet Med. 2017;34:27-36

30. Zhao J, Liu X, Zhang W. The effect of metformin therapy for preventing gestational diabetes mellitus in women with polycystic ovary syndrome: a meta-analysis. Exp Clin Endocrinol Diabetes. 2018: Epub ahead of print.

31. Vanky E, Stridsklev S, Heimstad R, et al. Metformin versus placebo from first trimester to delivery in polycystic ovary syndrome: a randomized controlled multicenter study. J Clin Endocrinol Metab. 2010;95:E448-55.

32. Moll E, Bossuyt PM, Korevaar JC, et al. Effect of clomifene citrate plus metformin and clomifene citrate plus placebo on induction of ovulation in women with newly diagnosed polycystic ovary syndrome: randomised double blind clinical trial. BMJ. 2006:332:1485.

33. Abu Hashim H, Foda O, Ghayaty E. Combined metforminclomiphene in clomiphene-resistant polycystic ovary syndrome: a systematic review and meta-analysis of randomized controlled trials. Acta Obstet Gynecol Scand. 2015;94:921-30.

34. Thessaloniki ESHRE/ASRM-Sponsored PCOS Consensus Workshop Group. Consensus on infertility treatment related to polycystic ovary syndrome. Hum Reprod. 2008;23:462-77.

35. Dickey RP, Taylor SN, Lu PY, et al. Effect of diagnosis. age. sperm quality. and number of preovulatory follicles on the outcome of multiple cycles of clomiphene citrate-intrauterine insemination. Fertil Steril. 2002;78:1088-95.

36. Wang R, Kim BV, van Wely M, et al. Treatment strategies for women with WHO group II anovulation: systematic review and networkmeta-analysis. BMJ. 2017;356:138.

37. Gadalla MA, Huang S, Wang R, et al. Effect of clomiphene citrate on endometrial thickness, ovulation, pregnancy and live birth 
in anovulatory women: systematic review and meta-analysis. Ultrasound Obstet Gynecol. 2018;51:64-76.

38. Brown J, Farquhar C. Clomiphene and other antioestrogens for ovulation induction in polycystic ovarian syndrome. Cochrane Database Syst Rev. 2016:12:CD002249.

39. Fields $E$, Chard J, James D, Treasure T. Guideline Development Group. Fertility (update): summary of NICE guidance. BMJ. 2013;346:林0

40. Practice Committee of the American Society for Reproductive Medicine. Use of clomiphene citrate in infertile women: a committee opinion. Fertil Steril. 2013;100:341-8.

41. Roque $\mathrm{M}$, Tostes $\mathrm{AC}$, Valle $\mathrm{M}$, et al. Letrozole versus clomiphene citrate in polycystic ovary syndrome: systematic review and meta-analysis. Gynecol Endocrinol. 2015;31:917-21.

42. Hu S, Yu Q, Wang Y, et al. Letrozole versus clomiphene citrate in polycystic ovary syndrome: a meta-analysis of randomized controlled trials. Arch Gynecol Obstet. 2018;297(5):1081-8

43. Legro RS, Brzyski RG, Diamond MP, et al. NICHD Reproductive Medicine Network. Letrozole versus clomiphene for infertility in the polycystic ovary syndrome. N Engl J Med. 2014;371:119-29.

44. Franik S, Kremer JA, Nelen WL, Farquhar C. Aromatase inhibitors for subfertile women with polycystic ovary syndrome. Cochrane Database Syst Rev. 2014;24:CD010287.

45. Jirge PR, Patil RS. Comparison of endocrine and ultrasound profiles during ovulation induction with clomiphene citrate and letrozole in ovulatory volunteer women. Fertil Steril. 2010;93:174-83.

46. Seow KM, Juan CC, Hwang JL, Ho LT. Laparoscopic surgery in polycystic ovary syndrome: reproductive and metabolic effects. Semin Reprod Med. 2008:26:101-10.

47. Hueb CK, Dias-Junior JA, Abrão MS, Filho EK. Drilling: medical indications and surgical technique. Rev Assoc Med Bras. 2015;61:530-5

48. Lebbi I, Ben-Temime R, Fadhlaoui A, Feki A. Ovarian drilling in PCOS: is it really useful? Front Surg. 2015;2:30.

49. Abu Hashim H, Foda O, El Rakhawy M. Unilateral or bilateral aparoscopic ovarian drilling in polycystic ovary syndrome: a meta-analysis of randomized trials. Arch Gynecol obstet. 2018:297:859-70.

50. Farquhar C, Brown J, Marjoribanks J. Laparoscopic drilling by diathermy or laser for ovulation induction in anovulatory polycystic ovary syndrome. Cochrane Database Syst Rev. 2012;6:CD001122.

51. Abu Hashim H. Predictors of success of laparoscopic ovarian drilling in women with polycystic ovary syndrome: an evidencebased approach. Arch Gynecol Obstet. 2015:291:11-8.

52. Mercorio F, Mercorio A, Di Spiezio Sardo A, et al. Evaluation of ovarian adhesion formation after laparoscopic ovarian drilling by second-look minilaparoscopy. Fertil Steril. 2008;89:1229-33.

53. Api M. Is ovarian reserve diminished after laparoscopic ovarian drilling? Gynecol Endocrinol. 2009;25:159-65.

54. Homburg R, Hendriks ML, König TE, et al. Clomifene citrate or low-dose FSH for the frst-line treatment of infertile women with anovulation associated with polycystic ovary syndrome: a prospective randomized multinational study. Hum Reprod. 2012;27:468-73.
55. Casadei L, Puca F, Emidi E, et al. Sequential low-dose step-up and step-down protocols with recombinant follicle-stimulating hormone in polycystic ovary syndrome: prospective comparison with step-down protocol. Arch Gynecol Obstet. 2012;286: $1291-7$

56. Hugues JN, Soussis J, Calderon I, et al. Recombinant LH Study Group. Does the addition of recombinant LH in WHO group II anovulatory women over-responding to FSH treatment reduce the number of developing follicles? A dose-finding study. Hum Reprod. 2005;20:629-35.

57. Platteau P, Andersen AN, Balen A, et al. Menopur Ovulation Induction (MOI) Study Group. Similar ovulation rates, but different follicular development with highly purified menotrophin compared with recombinant FSH in WHO Group II anovulatory infertility: a randomized controlled study. Hum Reprod. 2006;21:1798-804.

58. Weiss NS, Nahuis M, Bayram N, et al. Gonadotropins for ovulation induction in women with polycystic ovarian syndrome. Cochrane Database Syst Rev. 2015;9:CD010290.

59. Nahuis M, van der Veen F, Oosterhuis J, et al. Review of the safety. efficacy. costs and patient acceptability of recombinant follicle-stimulating hormone for injection in assisting ovulation induction in infertil. Int I Womens Health. 2010:1:205-11.

60. Bordewijk EM, Nahuis M, Costello MF, et al. Metformin during ovulation induction with gonadotrophins followed by timed intercourse or intrauterine insemination for subfertility associated with polycystic ovary syndrome. Cochrane Database Syst Rev. 2017;24:CD009090.

61. Heijnen EM, Eijkemans MJ, Hughes EG, et al. A meta-analysis of outcomes of conventional IVF in women with polycystic ovary syndrome. Hum Reprod Update. 2006;12:13-21.

62. Li HW, Lee VC, Lau EY, et al. Cumulativen live-birth rate in women with polycystic ovary syndrome or isolated polycystic ovaries undergoing in-vitro fertilisation treatment. J Assist Reprod Genet. 2014;31:205-11

63. Soave I, Marci R. Ovarian stimulation in patients in risk of OHSS. Minerva Ginecol. 2014;66:165-78

64. Al-Inany HG, Youssef MA, Aboulghar M, et al. Gonadotrophinreleasing hormone antagonists for assisted reproductive technology. Cochrane Database Syst Rev. 2016;4:CD001750

65. Kolibianakis E, Schultze-Mosgau A, Schroer A, et al. A lower ongoing pregnancy rate can be expected when GnRH agonist is used for triggering final oocyte maturation instead of hCG, in patients undergoing IVF with GnRH antagonists. Hum Reprod. 2005;20:2887-92

66. Griesinger G, Diedrich K, Devroey P, Kolibianakis EM. GnRHagonist for triggering final oocyte maturation in the GnRHantagonist ovarian hyperstimulation protocol: a systematic review and meta-analysis. Hum Reprod update. 2006;12:159-68.

67. Griesinger G, Kolibianakis EM, Papanikolaou EG, et al. Triggering of final oocyte maturation with gonadotropin releasing hormone agonist or human chorionic gonadotropin. Live birth after frozen-thawed embryo replacement cycles. Fertil Steril. 2007:88:616-21

68. Genazzani AD. Inositol as putative integrative treatment for PCOS.
Reprod Biomed Online. 2016;33:770-80

69. Papaleo E, Unfer V, Baillargeon JP, Chiu TT. Contribution of myo-inositol to reproduction. Eur J Obstet Gynecol Reprod Biol 2009; 147:120-3.

70. Laganà AS, Barbaro L, Pizzo A. Evaluation of ovarian function and metabolic factors in women afected by polycystic ovary syndrome after treatment with d-chiro-inositol. Arch Gynecol Obstet. 2015:291:1181-6.

71. Unfer V, Facchinetti F, Orrù B, et al. Myo-inositol efects in women with PCOS: a meta-analysis of randomized controlled trials. Endocr Connect. 2017;6:647-58.

72. Laganà AS, Rossetti P, Sapia F, et al. Evidence-based and patient-oriented inositol treatment in polycystic ovary syndrome: changing the perspective of the disease. Int J Endocrinol Metab. 2017; 22:e43695

73. Pizzo $\mathrm{A}$, Laganà AS, Barbaro L. Comparison between effects of myo-inositol and d-chiroinositol on ovarian function and metabolic factors in women with PCOS. Gynecol Endocrinol. 2014;30:205-8.

74. Venturella O, Mocciaro R, De Trana E, et al. Assessment of the modification of the clinical. endocrinal and metabolical profile of patients with PCOS syndrome treated with Myo-inositol. Minerva Ginecol. 2010;64:239-43.

75. Kamenov Z, Kolarov G, Gateva A, et al. Ovulation induction with myo-inositol alone and in combination with clomiphene citrate in polycystic ovarian syndrome patients with insulin resistance. Gynecol Endocrinol. 2015;31:131-5.

76. Gerli S, Papaleo E, Ferrari A, Di Renzo GC. Randomized, doubleblind placebocontrolled trial: effects of myo-inositol on ovarian function and metabolic factors in women with PCOS. Eur Rev Med Pharmacol Sci. 2007:11:347-54.

77. Genazzani AD, Prati A, Santagni S, et al. Differential insulin response to myo-inositol administration in obese polycystic ovary syndrome patients. Gynecol Endocrinol. 2012;28:969-73.

78. Genazzani AD, Santagni S, Rattighieri E, et al. Modulatory role of d-chiro-inositol (DCl) on $\mathrm{LH}$ and insulin secretion in obese pcos patients. Gynecol Endocrinol. 2014;30:438-43.

79. Pundir J, Psaroudakis D, Savnur P, et al. Inositol treatment of anovulation in women with polycystic ovary syndrome: a metaanalysis of randomised trials. BJOG. 2018;125:299-308.

80. Mendoza N, Pérez L, Simoncini T, Genazzani A. Inositol supplementation in women with polycystic ovary syndrome undergoing intracytoplasmicsperm injection: a systematic review and meta-analysis of randomized controlled trials. Reprod Biomed Online. 2017;35:529-35.

81. Facchinetti F, Bizzarri M, Benvenga S, et al. Results from the international consensus conference to on myo-inositol and dchiro-inositol in obstetrics and gynecology: the link between metabolic syndrome and PCOS. Eur J Obstet Gynecol Reprod Biol. 2015;195:72e6

82. Musi N, Hirshman MF, Nygren J, et al. Metformin increases AMPactivated protein kinase activity in skeletal muscle of subjects with type 2 diabetes. Diabetes. 2002;51:2074-81.

83. Genazzani AD, Shefer K, Della Casa D, et al. Modulatory effects of alpha-lipoic acid (ALA) administration on insulin sensitivity in obese PCOS patients. J Endocrinol Invest. 2018:41:583-90 